\title{
CORRIGENDUM
}

doi:10.1038/nature22318

\section{Corrigendum: Disentangling type 2 diabetes and metformin treatment signatures in the human gut microbiota}

Kristoffer Forslund, Falk Hildebrand, Trine Nielsen, Gwen Falony, Emmanuelle Le Chatelier, Shinichi Sunagawa, Edi Prifti, Sara Vieira-Silva, Valborg Gudmundsdottir, Helle Krogh Pedersen, Manimozhiyan Arumugam, Karsten Kristiansen, Anita Yvonne Voigt, Henrik Vestergaard, Rajna Hercog, Paul Igor Costea, Jens Roat Kultima, Junhua Li, Torben Jørgensen, Florence Levenez, Joël Dore, MetaHIT consortium, H. Bjørn Nielsen, Søren Brunak, Jeroen Raes, Torben Hansen, Jun Wang, S. Dusko Ehrlich, Peer Bork \& Oluf Pedersen

Nature 528, 262-266 (2015); doi:10.1038/nature15766

In the Supplementary Information to this Letter, data from two previous studies were used in the meta-analysis. However, the unit conversions used to make the data comparable were inconsistent for two of the included phenotype measures. Although this error does not affect the data used to generate the conclusions of the Letter, it might affect follow-up studies using the glycated haemoglobin (HbA1c) and serum insulin phenotypes. A revised Supplementary Table 1 ('phenotypes' tab) and corrected cohort summary statistics in Supplementary Tables 2 ('metadata' tab) and 12 ('metadata differences' tab) are provided in Supplementary Information to this Corrigendum.

In addition, in the Supplementary Information on page 6, the sentence "For clinically relevant markers, we found glycated haemoglobin A1c (HbA1c), reflecting long-term blood glucose levels, to be lowest in MHD T2D and highest in SWE ND CTRL samples $\left(P<2 \times 10^{-16}\right)$. Fasting plasma glucose levels were highest in CHN T2D patients and lowest in CHN ND CTRL, compared to corresponding SWE \& MHD samples $\left(P=5.6 \times 10^{-4}\right)$." should have read "For clinically relevant markers, we found fasting plasma glucose levels were highest in $\mathrm{CHN}$ T2D patients and lowest in CHN ND CTRL, compared to corresponding SWE \& MHD samples $\left(P=5.6 \times 10^{-4}\right)$." On page 18 , the sentence "To convert $\mathrm{mU} / \mathrm{l}$ to $\mathrm{pmol} / \mathrm{l}$ for insulin levels a factor of 6.0 was used." should have read "To convert $\mathrm{mU} / \mathrm{l}$ to $\mathrm{pmol} / \mathrm{l}$ for insulin levels a factor of 6.945 was used."

We would like to thank the readers who brought these issues to our attention. The original Letter has not been corrected online.

Supplementary Information is available in the online version of the Corrigendum. 